\title{
Dielectric Response of 1,1-Difluorosumanene Caused by an In-Plane Motion
}

\author{
Minghong Li, ${ }^{[a]}$ JianYun Wu ${ }^{[b]}$ Kohei Sambe, ${ }^{[b]}$ Yumi Yakiyama, ${ }^{* a],[c]}$ Tomoyuki Akutagawa, ${ }^{[b],[d]}$ Takashi \\ Kajitani, ${ }^{[e]}$ Takanori Fukushima, ${ }^{[f],[g]}$ Kazunari Matsuda, ${ }^{[h]}$ and Hidehiro Sakuraii[a,[c]
}

[a] Minghong Li, Prof. Dr. Yumi Yakiyama, Prof. Dr. Hidehiro Sakura

Division of Applied Chemistry, Graduate School of Engineering

Osaka University

2-1 Yamadaoka, Suita, Osaka 565-0871, Japan

E-mail: yakiyama@chem.eng.osaka-u.ac.jp

[a] Prof. Dr. Yumi Yakiyama, Prof. Dr. Hidehiro Sakurai

Innovative Catalysis Science Division, Institute for Open and Transdisciplinary Research Initiatives (ICS-OTRI)

Osaka University

2-1 Yamadaoka, Suita, Osaka 565-0871, Japan

E-mail: yakiyama@chem.eng.osaka-u.ac.jp

[c] Dr. J. Wu, K. Sambe, Prof. Dr. T. Akutagawa

Graduate School of Engineering,

Tohoku University

6-6 Aramakiazaaoba, Aoba-ku, Sendai 980-8579, Japan

[d] Prof. Dr. T. Akutagawa

Institute of Multidisciplinary Research for Advanced Materials (IMRAM)

Tohoku University

2-1-1 Katahira, Aoba-ku, Sendai 980-8577, Japan

[e] Dr. T. Kajitani

Open Facility Development Office, Open Facility Center

Tokyo Institute of Technology

4259 Nagatsuta, Midori-ku, Yokohama 226-8503, Japan

[f] Prof. Dr. T. Fukushima

Laboratory for Chemistry and Life Science, Institute of Innovative Research

Tokyo Institute of Technology

4259 Nagatsuta, Midori-ku, Yokohama 226-8503, Japan

[g] Prof. Dr. T. Fukushima

Department of Chemical Science and Engineering

School of Materials and Chemical Technology, Tokyo Institute of Technology

4259 Nagatsuta, Midori-ku, Yokohama 226-8503, Japan

[h] Prof. Dr. K. Matsuda

Institute of Advanced Energy, Kyoto University

Gokasho, Uji, Kyoto 611-0011, Japan

Supporting information for this article is given via a link at the end of the document

\begin{abstract}
We have designed and synthesized a new curved $\pi-$ conjugated molecule, 1,1-difluorosumanene (1), which possesses two fluorine atoms on the same benzylic carbon of unsubstituted sumanene, producing a large dipole moment along the in-plane direction. Thermal analyses, variable-temperature X-ray diffraction, and IR measurements indicated a $120^{\circ}$ in-plane motion of 1 in the stacking columns, which did not cause any phase transition in the crystalline state. Indeed, dielectric measurements on powder and single-crystal forms of 1 showed that both the real $\left(\varepsilon_{1}\right)$ and imaginary $\left(\varepsilon_{2}\right)$ parts of the dielectric constant were enhanced above $\sim 360 \mathrm{~K}$ at $1 \mathrm{MHz}$ with a Debye-type dielectric relaxation, confirming that 1 underwent a pendulum fluctuation induced by the external electric field.
\end{abstract}

\section{Introduction}

Organic-molecule-based crystalline dielectric materials have recently attracted widespread attention from chemists wishing to develop new organic-electronic devices. ${ }^{[1]}$ In designing such materials, a molecular motion induced by an external dielectric field is required to maximize the polarization effect in the materials to realize a large dielectric constant. In general, molecular motions in rotor-like structures, ${ }^{[2]}$ in-plane motions of planar units in $\pi$-stacking columns ${ }^{[3,4]}$ or supramolecular units, ${ }^{[5]}$ and dynamic motions of polar globular molecules ${ }^{[6]}$ are often investigated (Figure 1a). Such molecular motions in the solid state can be roughly categorized into four types: free rotation, rotational diffusion, jump rotation, and libration. ${ }^{[7]}$ For example, perdeuterated benzene trapped in tri-ortho-thymotide shows an in-plane rotation (almost a $2 \pi / 6$ jump model), together with restricted libration of the molecular plane in the temperature range $96-293 \mathrm{~K}^{\left[{ }^{[8]}\right.}$ Such detailed aspects of the molecular motions are significantly affected by the molecular structure, in particular, the intermolecular interactions involved in the system. The molecular motions of curved- $\pi$ aromatics and their supramolecular complexes, especially those containing $\mathrm{C}_{60}$ fullerenes, often show characteristic smoothness. For example, $\mathrm{C}_{60}$ is known to rotate isotropically in the solid state. ${ }^{[9]}$ Its smooth motion is also maintained in a system in which $\mathrm{C}_{60}$ is contained in a finite single-wall carbon nanotube, even at low temperatures in the single-crystalline state. This results from nondirectional van der Waals interactions between $\mathrm{C}_{60}$ and the tubular molecule, which has an inflection-free surface. ${ }^{[10]}$ Such 

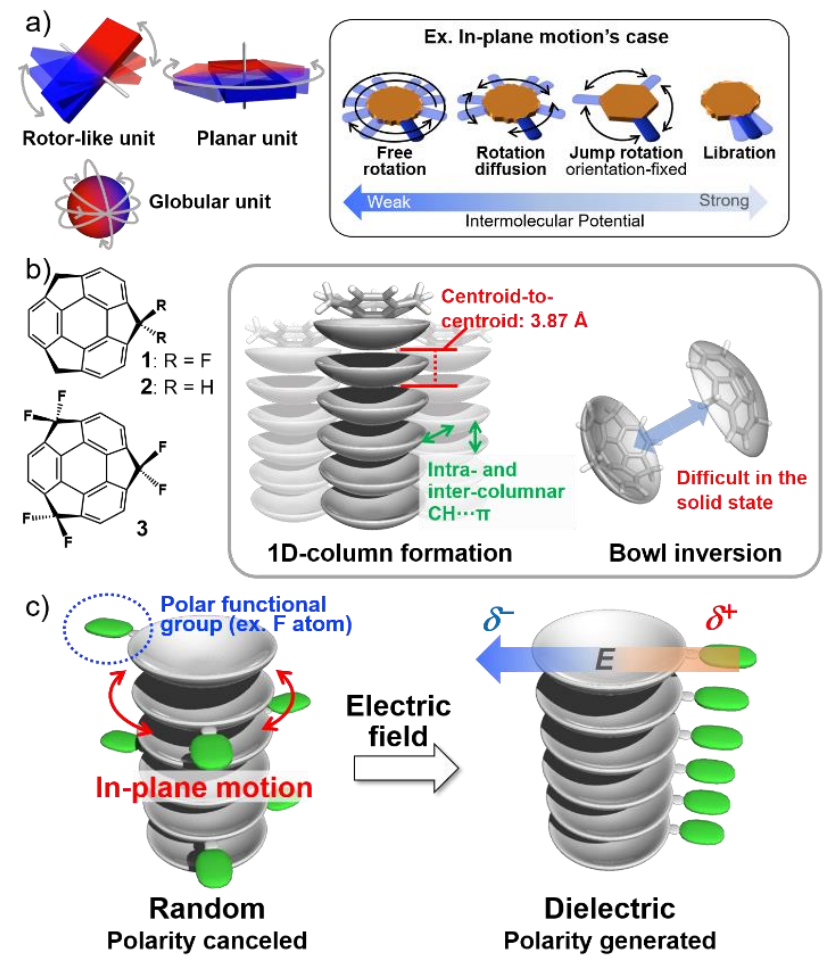

Figure 1. a) Typical molecular motions in organic-molecule-based crystalline dielectric materials. b) Molecular structures of 1, 2, and 3. b) Schematic diagrams of 1D-column formation and bowl inversion of 2. c) Model structures of in-plane motion of the bowl exhibiting a dielectric response in an electric field.

examples show that curve-to-curve contacts in curved- $\pi$ aromatics can afford smooth molecular motions in the solid state.

Sumanene (2) is a representative example of a buckybowl ${ }^{[11]}$ that has unique properties derived from its unusual bowl shape (Figure 1b). ${ }^{[12]}$ In particular, 2 forms unidirectionally arranged $\pi-$ stacking columns in the solid state that show an anisotropic electron mobility along the stacking column ${ }^{13]}$ and a large Seebeck co-efficient at right angles to the column; ${ }^{[14]}$ moreover, 2 can operate as a liquid-crystalline mesogen. ${ }^{[15]}$ This background clearly shows that the sumanene skeleton has considerable potential as a core for functional materials. It is also known that $\mathbf{2}$ shows bowl inversion along the dipole in the solution state. Such bowl flipping is attractive as a potential switching trigger for control of physical functions. However, its activation energy in the solid state is too high because of the formation of tightly-packed 1D-columns; consequently, the application of the bowl-flipping motion of sumanene was first realized in a sulfur analogue, 1,4,7-trithiasumanene, which possesses much a shallower bowl than $2 .{ }^{[16]}$

In this context, we focused on the in-plane motion of $\mathbf{2}$ in its columnar form, in which convex-concave-type curve-to-curve contacts exist, and we attempted to use this motion to bring out some physical functions. It had already been revealed that the dominant intermolecular interaction present in the crystal packing of sumanene are inter- and intracolumnar weak $\mathrm{CH}-\pi$ interactions between benzylic $\mathrm{sp}^{2}$ carbons and the $\pi-b o w l$, rather than intracolumnar $\pi-\pi$ interactions (the distance between the two centroids of the bottom six-membered rings in a stacking column of 2 is $3.87 \AA$ ). ${ }^{[1]]}$ The presence of this $\pi-\pi$ interaction that is weaker than that in a conventional planar $\pi-$ conjugated molecule is expected to provide a higher flexibility for in-plane motion in the column. Meanwhile, the presence of a $\mathrm{CH}-\mathrm{T}$ interaction should contribute to stabilization of the molecular packing, as occurs in a Saturn-like $\mathrm{C}_{60} /$ macrocycle complex, ${ }^{[18]}$ increasing the activation energy for in-plane motion. Clarification of such opposing effects in the crystal packing of 2 would be significant for further applications of functional sumanene derivatives, especially those utilizing their in-plane motions

We therefore focused on a dielectric response that might be induced through the introduction of polar substituents as a probe for the ease of in-plane motion (Figure 1c). Previously reported 1,1,4,4,7,7-hexafluorosumanene (3), which contains two fluorine atoms on each of the three benzylic carbons, was an important inspiration for this purpose. ${ }^{[19]}$ Single-crystal X-ray analysis revealed that the crystal packing of $\mathbf{3}$ is isostructural to $\mathbf{2}$, indicating that, because of the small size of the fluorine atom, the introduction of fluoro groups onto the sumanene skeleton does not significantly affect its packing pattern. On the basis of this assumption, we have designed and synthesized 1,1difluorosumanene (1), which contains two fluorine atoms on one benzylic carbon of $\mathbf{2}$, inducing a strong dipole along the in-plane direction. As we had expected, a single crystal of 1 showed a molecular packing isostructural to that of $\mathbf{2}$ and $\mathbf{3}$. In addition, a remarkable anisotropy of the temperature-dependent dielectric response of $\mathbf{1}$, attributable to its in-plane motion in the stacking column as a result of the two competing interactions, was confirmed from the dielectric spectra of a single-crystalline sample.

\section{Results and Discussion}

1,1-Difluorosumanene 1 was synthesized by a modified form of a reported procedure (Scheme 1). ${ }^{[19]}$ Protection of the carbonyl group of previously reported sumanenen-1-one ${ }^{[20]}$ as a dithioacetal by treatment with butane-1,4-dithiol the presence of $\mathrm{BF}_{3} \cdot \mathrm{OEt}_{2}$ gave 4 in $95 \%$ yield. Fluorination of $\mathbf{4}$ with $250 \mathrm{~mol} \%$ of NBS and 2.5 mol\% HF.pyridine at $-30{ }^{\circ} \mathrm{C}$ gave 1 in $77 \%$ yield.

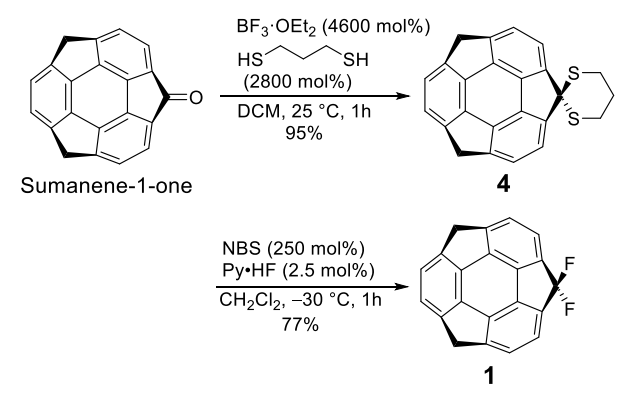

Scheme 1. Synthesis of 1,1-difluorosumanene 1

To investigate the effect of introducing two fluorine atoms onto the sumanene skeleton, we first evaluated the structural and the electronic properties of $\mathbf{1}$ by DFT calculations at the B3LYP/6-31G(d,p) level of theory (Table 1 and Figure 2). ${ }^{[21]}$ The optimized structure of $\mathbf{1}$ showed the presence of a bowl that is slightly deeper $(1.16 \AA)$ than that of unsubstituted sumanene 2 $(1.12 \AA)$, but shallower than that of hexafluorosumanene 3 (1.23 
Table 1. Structural parameters of 1, 2 and 3 . The values in parentheses are those calculated at the B3LYP/6-31G(d,p) level of theory. ${ }^{[21]}$ The figure below shows the definitions of the bowl depth and POAV angle.

\begin{tabular}{cccc}
\hline Compound & $\mathbf{1}$ & $\mathbf{2}$ & $\mathbf{3}$ \\
\hline Bowl depth $(\AA)$ & $1.16(1.15)$ & $1.11^{[17]}(1.12)$ & $1.24^{[19]}(1.23)$ \\
POAV angle $\left({ }^{\circ}\right)$ & $8.9(9.0)$ & $8.9^{[17]}(8.7)$ & $9.4^{[19]}(9.3)$ \\
Dipole moment $(\mathrm{D})$ & $(3.62)$ & $(1.94)$ & $(1.22)$ \\
\hline
\end{tabular}
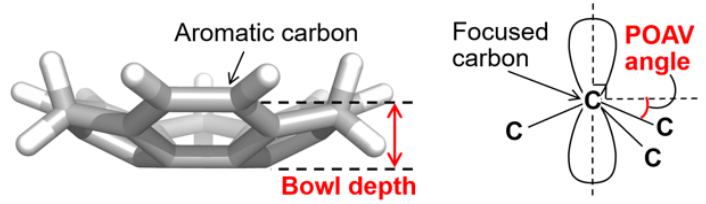
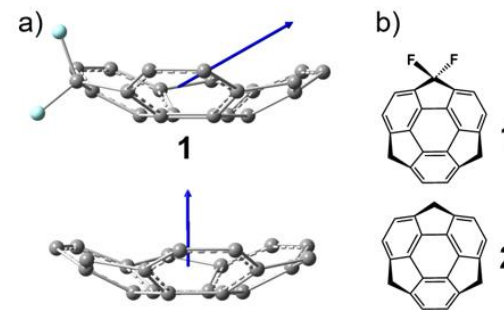

2

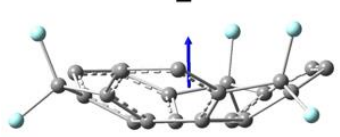

3

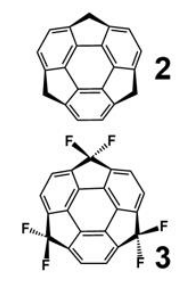

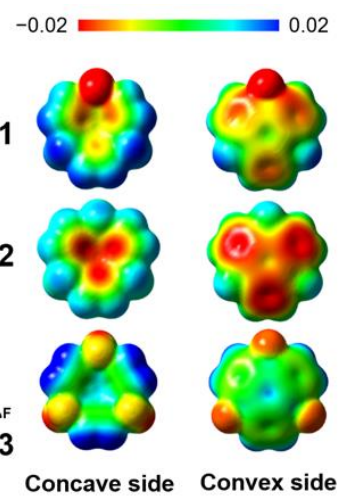

Figure 2. Electronic structures of 1, 2, and $\mathbf{3}$ from DFT calculation at the B3LYP/6-31G(d,p) level of theory. a) Calculated dipole-moment directions of 1, 2, and 3. Gray: C, pale-blue: F. Hydrogen atoms are omitted for clarity. b) Electrostatic potential maps for $\mathbf{1}, \mathbf{2}$, and $\mathbf{3}$.

$\AA$ ). This was supported by the larger $\pi$-orbital axis vector (POAV) angle of $\mathbf{1}\left(8.9^{\circ}\right)$ compared with that of $2\left(8.7^{\circ}\right)$; this is a useful parameter for discussing the curvature of curved- $\pi$ aromatics. ${ }^{[22]}$ The electrostatic potential of $\mathbf{1}$ clearly showed the presence of an electronegative region due to presence of the two fluorine atoms on one benzylic carbon atom, which induce a high degree of polarization of $\mathbf{1}$ (Figure 2a). As a result, $\mathbf{1}$ has the largest dipole moment among 1,2 , and 3 , with a value of 3.62 D, whereas in $\mathbf{2}$ and $\mathbf{3}$, the in-plane dipoles tend to cancel one another out because of the high degree of structural symmetry of these molecules (Figure 2b). As expected, the presence of highly electronegative fluorine atoms causes a significant decline in both HOMO and LUMO levels compared with those of 2, whereas they are higher than those of $\mathbf{3}$ [see the Supplementary Information (SI); Figure S1]. This electronic perturbation resulting from the introduction of fluorine atoms was well reflected in the results of UV-vis spectroscopy and cyclic voltammetry (CV) experiments. The UV-vis spectrum of 1 (SI; Figure S2) showed an absorption maximum at $273 \mathrm{~nm}$, together with a broad peak at about $350 \mathrm{~nm}$ that corresponded to the HOMO-LUMO transition; the positions of both peaks were between those of the corresponding peaks for 2 and $3 .^{[19]} \mathrm{CV}$ data for $\mathbf{1}$ in MeCN with tetrabutylammonium perchlorate as a supporting electrolyte showed a positive shift in the reduction a)
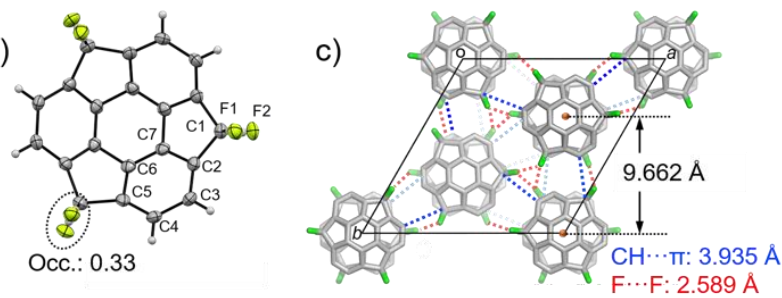

b)

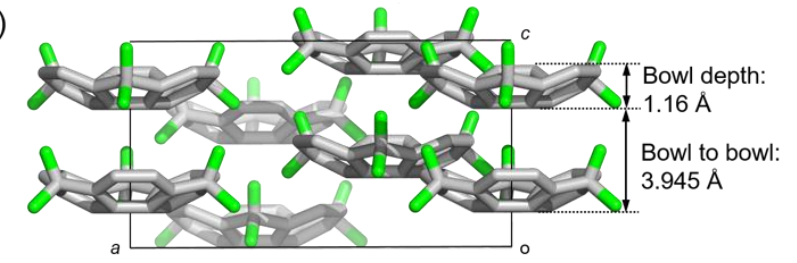

Figure 3. Crystal structures of 1. a) Thermal displacement ellipsoid plot of $\mathbf{1}$ in the crystal structure at $50 \%$ probability. Occupancy factors of F1 and F2 are 0.33 each. b) View from the $a$ axis. c) View from the $c$ axis. Blue dotted lines: $\mathrm{CH} \cdots \pi$ interactions; red dotted lines: F-F interactions. Gray: C; light-green: F. Hydrogen atoms are omitted for clarity.

peak in comparison with that of 2 . Irreversible reduction waves were observed at the peak potentials $E_{p}^{\text {red1 }}=-2.37 \mathrm{~V}$ and $E_{p}{ }^{\text {red2 }}$ $=-2.58 \mathrm{~V}$ vs. the ferrocene/ferrocenium couple $\left(\mathrm{Fc}^{0} / \mathrm{Fc}^{+}\right)(\mathrm{SI}$; Figure S3). This indicated that $\mathbf{1}$ has a stronger electronaccepting nature than $2(-2.60 \mathrm{~V})$. $^{[23]}$

The structural details of $\mathbf{1}$ were confirmed by a single-crystal X-ray diffraction experiment at $100 \mathrm{~K}$. Suitable single crystals of 1 were prepared by slow evaporation of a chloroform solution of 1. Despite 1 having lower molecular symmetry than $C_{3 v^{-}}$ symmetric 2 , crystals of $\mathbf{1}$ show the same packing pattern as that observed in crystals of $\mathbf{2}$, due to the small size of the fluorine atom. A disorder of the fluorinated moiety was observed in its occupancy factor of 0.33 (Figure 3a), which indicated a canceling of the dipole moment of $\mathbf{1}$ in the in-plane direction. The sumanene skeleton of $\mathbf{1}$ has a bowl structure with a bowl depth of $1.16 \AA$ and a POAV angle of $9.0^{\circ}$, which is slightly deeper than that of unsubstituted $2(1.11 \AA$; Table 1$) .^{[17]}$ The trends in these structural parameters are well-matched by the simulated values. In the packing structure, the bowl-to-bowl distance of $\mathbf{1}$ is $3.945 \AA$; this is defined as the distance between the two centroids of the six-membered rings at the bottom of adjacent sumanene skeletons in a stacking column (Figure $3 \mathrm{~b}$ ). This value was longer than that for $2(3.790 \AA),{ }^{[17]}$ probably due to the deeper bowl depth of $\mathbf{1}$ compared with $\mathbf{2}$. All the columns were connected to each other through $\mathrm{CH} \cdots \pi$-type interactions (3.935 $\AA$ ) between benzylic carbon atoms and neighboring aromatic carbon atoms with a partial contribution from $F$... F interactions $(2.589 \AA)$ to stabilize the packing structure (Figure 3c). Notably, the distance between the two closest adjacent column axes was $9.662 \AA$ (Figure 3c), whereas the corresponding distances in crystals of 2 and 3 are $9.570 \AA$ and $9.852 \AA$, respectively.

Because the expected stacking structure was successfully obtained, thermal analyses were performed to confirm the stability of the material and to identify the presence of any thermal phase transitions (SI; Figure S4). However, no clear peak attributable to a phase transition was observed, although specific-heat measurements showed a clear peak at around 350 $\mathrm{K}$ (SI; Figure S5), indicating the presence of some structural motion that did not affect to overall molecular packing. 


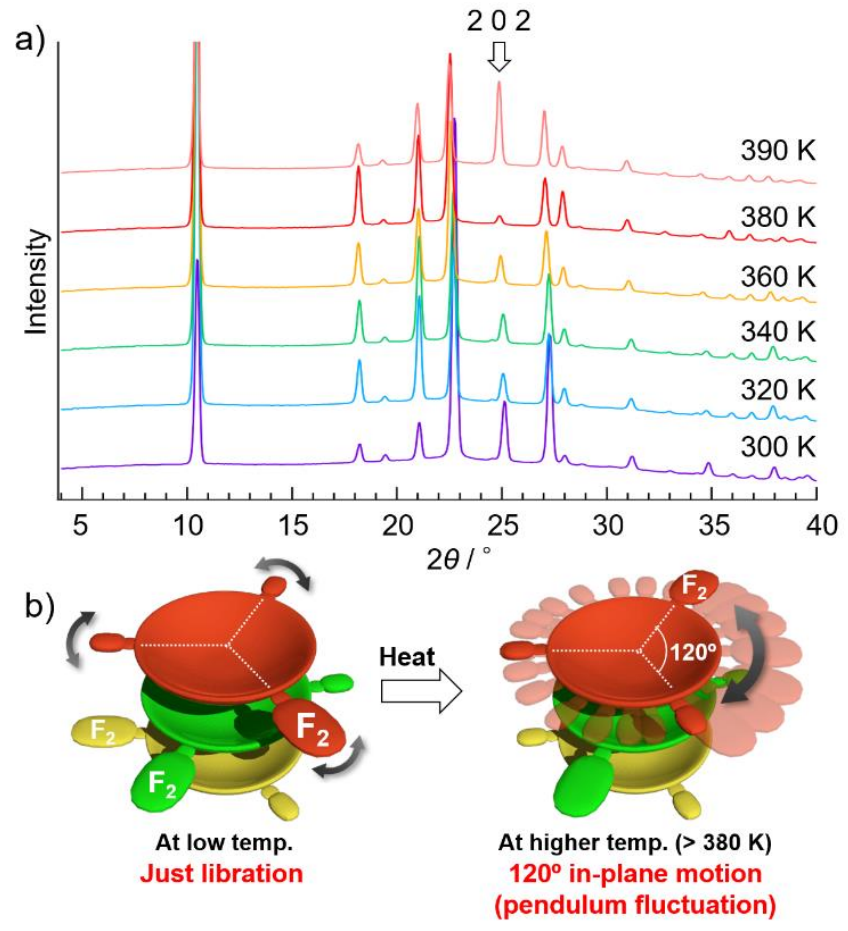

Figure 4. Thermal effects on the molecular motions of 2 in the solid state. a) TD-PXRD patterns of powdered crystalline 1. The small arrow indicates a peak that shows a change in intensity at $380 \mathrm{~K}$. b) Schematic representation of temperature-dependent molecular motion in the stacking column of $\mathbf{1}$. The white dotted line shows that the molecular orientation (the position of the three benzylic carbons) is retained in the crystal.

The effect of temperature on the structure was also evaluated by variable-temperature powder X-ray diffraction analysis (VT-PXRD) (Figure 4). Although no significant peak shift indicating a marked change in packing structure was observed on heating, a sudden change in peak intensity at around $2 \theta=25^{\circ}$, corresponding to 202 reflection, was observed at $380 \mathrm{~K}$ (SI; Figure S6a). This temperature is almost the same as that for the apparent peak observed in the heat-capacity measurement. The structural analysis based on the simulated pattern generated from the single-crystal data revealed that the crystal plane corresponding to the diffraction peak exits to cut the intracolumnar interactions (SI; Figure S6b). Therefore, this sudden peak sharpening indicates a weakening of the intermolecular interactions on heating, followed by peak equalization resulting from much faster molecular motion. We also collected diffraction data for a single crystal of 1 at $413 \mathrm{~K}$ (SI; Figure S7). These diffraction data revealed a slightly larger cell size than that observed at $100 \mathrm{~K}$, whereas all the bond lengths of $\mathbf{1}$ and the bowl depth were almost unchanged (SI; Figures S7 and S8). The cell-parameter magnification was reflected by increases in the intercolumnar distance (9.662 to $9.741 \AA$ ) and the bowl-to-bowl distance (3.945 to $4.085 \AA$ ), indicating the presence of weaker intermolecular interactions. Heating also caused a magnification of the displacement parameters of each atom and the long-axis directions of their thermal ellipsoids correspond to the in-plane motion (SI; Figure S9). However, no apparent rotational disorder was observed. These results strongly suggest that the focusing molecular motion at high temperatures involves an in-plane motion with retention of specific molecular orientations, namely a $120^{\circ}$ in- plane pendulum fluctuation in which all the benzylic C- $\mathrm{H}$ and $\mathrm{C}-$ $\mathrm{F}$ bonds continue to have the same types of interactions as those in the low-temperature structure (Figure 4b). Indeed, the intermolecular interactions in the system at $413 \mathrm{~K}$ as visualized by an energy-framework analysis using the CrystalExplorer program, indicated the presence of weak intercolumnar interactions, together with a much stronger intracolumnar interaction (SI; Figure S9). ${ }^{[2]}$ This structural feature of $\mathbf{1}$ supports the assumption that the intracolumnar $\pi-\pi$ and $\mathrm{CH}-\pi$ interactions might be the main cause of the observed hindered motion.

This was further investigated by the variable-temperature IR method (SI; Figure S10). Absorption data for a $\mathrm{KBr}$ pellet containing 1 placed on a purpose-built heater showed a slight peak broadening on heating. In addition, a distinct peak spread of around $1030-1200 \mathrm{~cm}^{-1}$, corresponding to a C-F stretching mode, showed a slight shift to a lower-wavenumber region at higher temperatures above $100{ }^{\circ} \mathrm{C}$. Notably, another band at around $780-810 \mathrm{~cm}^{-1}$, attributable to a $\mathrm{C}-\mathrm{H}$ out-of-plane bending mode, also similarly shifted to a lower wavenumber. These observations indicated that the molecular motions changed at about $100{ }^{\circ} \mathrm{C}$, although the overall intermolecular interactions remained at higher temperatures. This assumption matches the discussion derived from the thermal analyses and TD-XRD results.

With clear proof that $\mathbf{1}$ shows dynamic motion in the crystalline state on thermal activation, dielectric measurements were first performed on a compressed pellet of powdered crystalline $\mathbf{1}$ and its hexafluoro analogue $\mathbf{3}$ at several frequencies (SI; Figures $S 11$ and S12). In the case of 1, both real $\left(\varepsilon_{1}\right)$ and imaginary $\left(\varepsilon_{2}\right)$ parts of the dielectric constant were enhanced at above $\sim 360 \mathrm{~K}$ at $1 \mathrm{MHz}$, with a Debye-type dielectric relaxation (SI; Figure S11). This temperature range was in reasonable agreement with the other experimental observations. The $\ln (T)-T_{\mathrm{p} 1}{ }^{-1}$ plots of $\mathbf{1}$ indicated a linear correlation (SI; Figure S13), where $T_{\mathrm{p} 1}$ is the dielectric $\varepsilon_{1}$-peak and the $T$-value is the relaxation time of the inverse of the measured $f$-values of $T=1 /(2 \pi f)$, suggesting an activation energy for the thermally activated motion of 1 of $E_{\mathrm{a}}=65 \mathrm{~kJ} / \mathrm{mol}$. However, no clear dielectric response was observed in highly symmetric 3 (SI; Figure S12) because of cancelation of the dipole along the in-plane direction.

To obtain a more precise relationship between the molecular motions and the dielectric nature of 1 , we subjected a large single crystal of $\mathbf{1}$ to dielectric measurements by the twoelectrode method (Figures $5 \mathrm{a}$ and SI, S14). The m-stacking direction of crystal $\mathbf{1}$ along the $c$ axis (the red arrow direction in Figure $5 \mathrm{a}$ ) showed no clear dielectric response (Figures $5 \mathrm{~b}$ and 5c) In contrast, the direction vertical to the $\pi$-stacking axis (blue arrow direction in Fig. 5a) showed clear $\varepsilon_{1}$ and $\varepsilon_{2}$ responses with a Debye-type dielectric relaxation, as observed in the single crystal of $\mathbf{1}$ (Figures $5 \mathrm{~d}$ and $5 \mathrm{e}$ ). The activation energy estimated from the Arrhenius plot of the relaxation time $T$ vs. $T_{\mathrm{p} 1}{ }^{-1}$ was 62 $\mathrm{kJ} / \mathrm{mol}$ for the dielectric relaxation, which is almost the same value as that obtained for the powdered crystalline sample ( $\mathrm{SI}$; Figure S13). These experimental results were consistent with the induction of an anisotropic dielectric response through a thermally activated in-plane motion of $\mathbf{1}$. Although of course it is impossible to make a direct comparison with previously reported charge-transfer-type $\pi$-planar stacking systems, the activation energy that we obtained is comparable to those of $\pi$-planar 

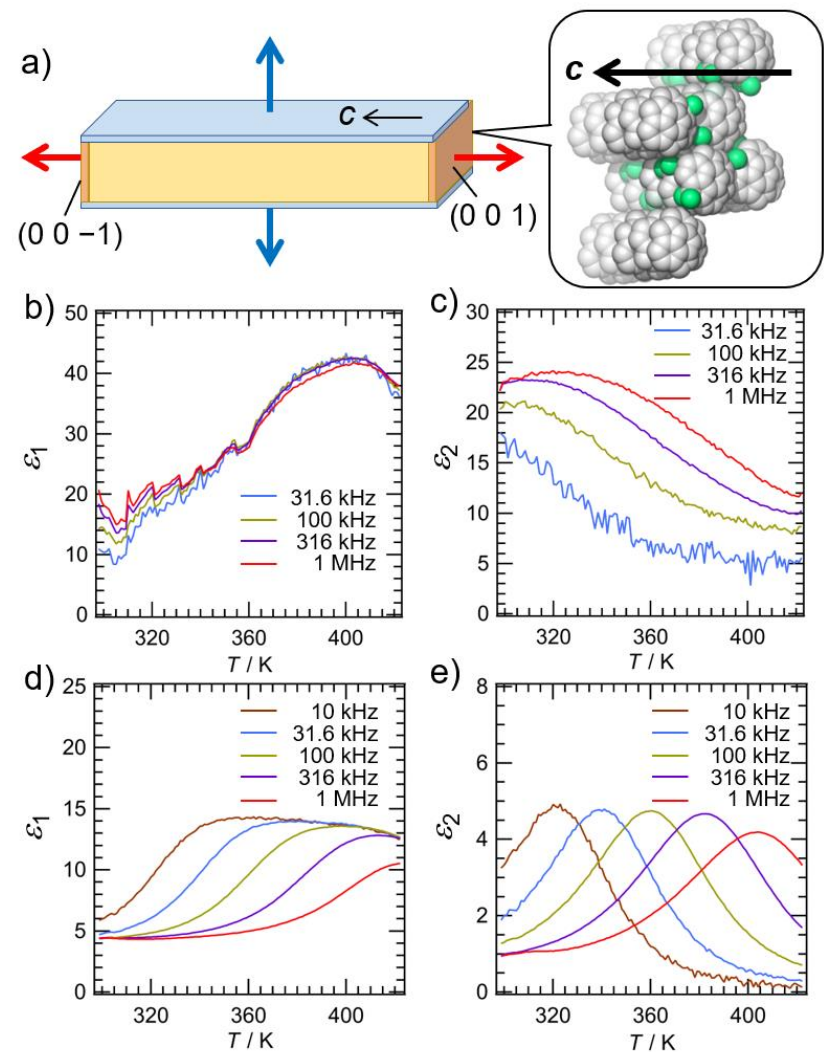

Figure 5. a) Schematic model of the relationship between the single-crysta shape of $\mathbf{1}$ and the applied electric field. b) to e) Temperature dependence of b), d) the real part $\left(\varepsilon_{1}\right)$ and c), e) the imaginary part $\left(\varepsilon_{2}\right)$ of the dielectric constant of 1 in a single-crystalline form measured at various frequencies. The direction of the electric field applied for $b$ ) and c): parallel to the $c$ axis; for $d$ ) and e): orthogonal to the $c$ axis.

systems $(48-56 \mathrm{~kJ} / \mathrm{mol})$ that can be described by an orderdisorder model. ${ }^{[4 b, c]}$ Our experimental results clearly indicate that the reduction of intracolumnar interactions, especially $\mathrm{CH} \cdots \pi$ interactions, is an important key to the development of new switching materials based on sumanene through utilization of its in-plane motion.

\section{Conclusion}

In conclusion, we have designed and synthesized a new curved $\pi$-conjugated molecule, 1,1-difluorosumanene $\mathbf{1}$, in which two fluorine atoms are present on one benzylic carbon of sumanene 2. DFT calculations showed that $\mathbf{1}$ has a larger dipole moment along the in-plane direction than those of unsubstituted 2 or symmetrical 1,1,4,4,7,7-hexafluorosumanene 3 . On heating, 1 exhibited a $120^{\circ}$ in-plane pendulum fluctuation that did not cause a phase transition in the crystalline state. These unique thermal properties of $\mathbf{1}$ realized an anisotropic dielectric response with a Debye-type dielectric relaxation in the singlecrystalline state, which also indicated an in-plane motion of $\mathbf{1}$ in the stacking columns. These results, focusing on the in-plane molecular motion in the $\pi$-stacking column of a buckybowl, will help to provide a better understanding of the dynamics of solidstate curved- $\pi$ systems and will accelerate their application in functional materials.

\section{Acknowledgements}

This work was supported by Grant-in-Aid for Scientific Research on Innovative Areas " $\pi$ Space Figuration" (No. JP26102002), Grant-in-Aid for Transformative Research Areas "Science of 2.5 Dimensional Materials" (Nos. JP21H05232, JP21H05233, JP21H05235), JSPS KAKENHI (JP19H00912, JP2OH00400), and Dynamic Alliance for Open Innovation Bridging Human, Environment and Materials (No. 20214025). The X-ray diffraction studies of $\mathbf{1}$ by synchrotron radiation were performed at BL02B1 of SPring-8 (2020A0808) and at the Pohang Accelerator Laboratory (Beamline 2D-SMC), supported by POSTECH (2020-1st-2D-043). The theoretical calculations were performed at the Research Centre for Computational Science, Okazaki, Japan. Y.Y. is grateful for support from the program "Initiative for Realizing Diversity in the Research Environment", Osaka University and to Dr. Dohyun Moon (POSTECH, Korea) for his considerable help with the high-temperature single-crystal X-ray diffraction measurements. M.L. thanks the China Scholarship Council for support (ID: CSC 201708310115, CSC 201908050056). We thank Dr. X. Chen of the Northeast Petroleum University for fruitful discussions on the DFT calculations.

Keywords: buckybowls $\cdot$ crystal engineering $\cdot$ dielectric response $\bullet$ fluorine $\cdot$ sumanene

[1] J. Brebels, J. V. Manca, L. Lutsen, D. Vanderzande, W. Maes, J. Mater Chem. A 2017, 5, 24037

[2] (a) R. D. Horansky, L. I. Clarke, J. C. Price, T.-A. V. Khuong, P. D. Jarowski, M. A. Garcia-Garibay, Phys. Rev. B: Condens. Matter Mater. Phys. 2005, 72, 014302; (b) T. Akutagawa, H. Koshinaka, D. Sato, S. Takeda, S.-I. Noro, H. Takahashi, R. Kumai, Y. Tokura, T. Nakamura, Nat. Mater. 2009, 8, 342; (c) W. Li, C.-T. He, Y. Zeng, C.-M. Ji, Z.-Y. Du, W. Zhang, X.-M. Chen, J. Am. Chem. Soc. 2017, 139, 8086; (d) M. Tsurunaga, Y. Inagaki, H. Momma, E. Kwon, K. Yamaguchi, K. Yoza, W. Setaka. Org. Lett. 2018, 20, 6934

[3] J.-i. Ichikawa, N. Hoshino, T. Takeda, T. Akutagawa, J. Am. Chem. Soc 2015, 137, 13155 .

[4] (a) R. Bhowal, A. A. Balaraman, M. Ghosh, S. Dutta, K. K. Dey, D Chopra, J. Am. Chem. Soc. 2021, 143, 1024; (b) J. Harada, N Yoneyama, S. Sato, Y. Takahashi, T. Inabe, Cryst. Growth Des. 2019 19, 291; (c) J. Harada, M. Ohtani, Y. Takahashi, T. Inabe, J. Am. Chem. Soc. 2015, 137, 4477

[5] C. Shi, X. Zhang, Y. Cai, Y.-F. Yao, W. Zhang, Angew. Chem. 2015, 127, 6304; Angew. Chem. Int. Ed. 2015, 54, 6206.

[6] J. Harada, T. Shimojo, H. Oyamaguchi, H. Hasegawa, Y. Takahashi, K. Satomi, Y. Suzuki, J. Kawamata, T. Inabe, Nat. Chem. 2016, 8, 946.

[7] The Plastically Crystalline State: Orientationally Disordered Crystals (Ed.: J. N. Sherwood), Wiley, Chichester, 1979.

[8] A. E. Aliev, K. D. M. Harris, A. Mahdyarfar J. Chem. Soc., Faraday Trans. 1995, 91, 2017.

[9] P. A. Heiney, J. E. Fischer, A. R. McGhie, W. J. Romanow, A. M Denenstein, J. P. McCauley, Jr., A. B. Smith, III, D. E. Cox, Phys. Rev. Lett. 1991, 66, 2911.

[10] (a) S. Sato, T. Yamasaki, H. Isobe, Proc. Natl. Acad. Sci. U. S. A. 2014 111, 8374; (b) T. Matsuno, Y. Kanai, S. Sato, Y. Maniwa, H. Isobe, Nat Commun. 2018, 9, 1907; (c) T. Matsuno, Y. Nakai, Y. Maniwa, M. Someya, S. Sato, H. Isobe, Chem. Asian. J. 2020, 15, 273.

[11] H. Sakurai, T. Daiko, T. Hirao, Science 2003, 301, 1878.

[12] M. Saito, H. Shinokubo, H. Sakurai, Mater. Chem. Front. 2018, 2, 635.

[13] T. Amaya, S. Seki, T. Moriuchi, K. Nakamoto, T. Nakata, H. Sakane, A. Saeki, T. Hirao, J. Am. Chem. Soc. 2009, 131, 408.

[14] (a) H. Kojima, M. Nakagawa, R. Abe, F. Fujiwara, Y. Yakiyama, H. Sakurai, M. Nakamura, Chem. Lett. 2018, 47, 524; (b) H. Kojima, R. 
Abe, F. Fujiwara, M. Nakagawa, K. Takahashi, D. Kuzuhara, H. Yamada, Y. Yakiyama, H. Sakurai, T. Yamamoto, M. Ikeda, M Nakamura, Mater. Chem. Front. 2018, 2, 1276.

[15] Y. Shoji, T. Kajitani, F. Ishiwari, Q. Ding, H. Sato, H. Anetai, T. Akutagawa, H. Sakurai, T. Fukushima, Chem. Sci. 2017, 8, 8405.

[16] S. Furukawa, J. Wu, M. Koyama, K. Hayashi, N. Hoshino, T. Takeda, Y. Suzuki, J. Kawamata, M. Saito, T. Akutagawa, Nat. Commun. 2021, 12 768.

[17] S. Mebs, M. Weber, P. Luger, B. M. Schmidt, H. Sakurai, S Higashibayashi, S. Onogi, D. Lentz, Org. Biomol. Chem. 2012, 10, 2218.

[18] Y. Yamamoto, E. Tsurumaki, K. Wakamatsu, S. Toyota, Angew. Chem. 2018, 130, 8331; Angew. Chem. Int. Ed. 2018, 57, 8199.

[19] B. M. Schmidt, B. Topolinski, S. Higashibayashi, T. Kojima, M. Kawano, D. Lentz, H. Sakurai, Chem. Eur. J. 2013, 19, 3282.

[20] T. Amaya, M. Hifumi, M. Okada, Y. Shimizu, T. Moriuchi, K. Segawa, Y.,o, T. Hirao, J. Org. Chem. 2011, 76, 8049.

[21] M. J. Frisch, G. W. Trucks, H. B. Schlegel, G. E. Scuseria, M. A. Robb, J. R. Cheeseman, G. Scalmani, V. Barone, G. A. Petersson, H. Nakatsuji, X. Li, M. Caricato, A. Marenich, J. Bloino, B. G. Janesko, R. Gomperts, B. Mennucci, H. P. Hratchian, J. V. Ortiz, A. F. Izmaylov, J. L. Sonnenberg, D. Williams-Young, F. Ding, F. Lipparini, F. Egidi, J. Goings, B. Peng, A. Petrone, T. Henderson, D. Ranasinghe, V. G. Zakrzewski, J. Gao, N. Rega, G. Zheng, W. Liang, M. Hada, M. Ehara, K. Toyota, R. Fukuda, J. Hasegawa, M. Ishida, T. Nakajima, Y. Honda, O. Kitao, H. Nakai, T. Vreven, K. Throssell, J. A. Montgomery, Jr., J. E. Peralta, F. Ogliaro, M. Bearpark, J. J. Heyd, E. Brothers, K. N. Kudin, V. N. Staroverov, T. Keith, R. Kobayashi, J. Normand, K. Raghavachari, A Rendell, J. C. Burant, S. S. lyengar, J. Tomasi, M. Cossi, J. M. Millam, M. Klene, C. Adamo, R. Cammi, J. W. Ochterski, R. L. Martin, K. Morokuma, O. Farkas, J. B. Foresman, D. J. Fox, Gaussian 09, Revision D.01, Gaussian, Inc., Wallingford CT, 2013.

[22] R. C. Haddon, J. Am. Chem. Soc. 1987, 109, 1676.

[23] P. Zanello, S. Fedi, F. F. de Biani, G. Giorgi, T. Amaya, H. Sakane, T. Hirao, Dalton Trans. 2009, 9192.

[24] M. J. Turner, S. Grabowsky, D. Jayatilaka, M. A. Spackman, J. Phys. Chem. Lett. 2014, 5, 4249. 


\section{Entry for the Table of Contents}

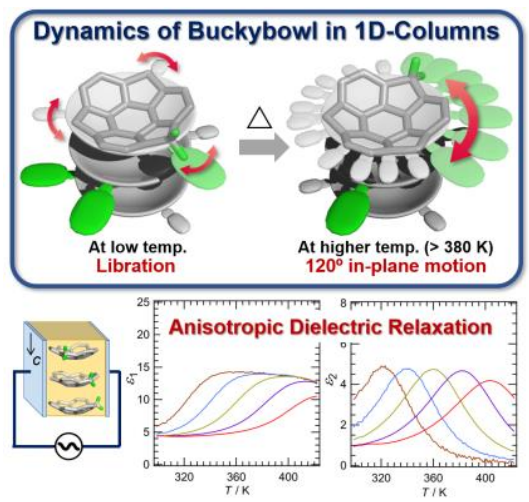

1,1-difluorosumanene, which possesses two fluorine atoms on the same benzylic carbon of unsubstituted sumanene, producing a large dipole moment along the in-plane direction was designed and investigated. It showed a $120^{\circ}$ in-plane pendulum motion in the stacking columns at above $380 \mathrm{~K}$. This dynamic motion in the solid state enabled the anisotropic dielectric relaxation induced by the external electric field.

Institute and/or researcher Twitter usernames: @Sakurailab_OU 